\title{
Fetal ECG Extraction from Maternal Abdominal ECG Using Neural Network
}

\author{
M. A. HASAN ${ }^{1}$, M. I. IBRAHIMY ${ }^{1}$, M. B. I. REAZ ${ }^{2}$ \\ ${ }^{1}$ Department of Electrical and Computer Engineering, International Islamic University Malaysia, Kuala Lumpur, Malaysia; \\ ${ }^{2}$ Department of Electrical, Electronic and Systems Engineering, University Kebangsaan Malaysia, Selangor, Malaysia. \\ Email: asraful.hasan@ieee.org, ibrahimy@iiu.edu.my,mamun.reaz@gmail.com
}

Received May 25 $5^{\text {th }}, 2009$; revised June $25^{\text {th }}, 2009$; accepted July $2^{\text {nd }}, 2009$.

\begin{abstract}
FECG (Fetal ECG) signal contains potentially precise information that could assist clinicians in making more appropriate and timely decisions during pregnancy and labor. The extraction and detection of the FECG signal from composite maternal abdominal signals with powerful and advance methodologies is becoming a very important requirement in fetal monitoring. The purpose of this paper is to illustrate the developed algorithms on FECG signal extraction from the abdominal ECG signal using Neural Network approach to provide efficient and effective ways of separating and understanding the FECG signal and its nature. The FECG signal was isolated from the abdominal signal by neural network approach with different learning constant value and momentum as well so that acceptable signal can be considered. According to the output it can be said that the algorithm is working satisfactory on high learning rate and low momentum value. The method appears to be exceedingly robust, correctly isolate the FECG signal from abdominal $E C G$.
\end{abstract}

Keywords: Neural Network, FECG, Abdominal ECG, Heart Rate

\section{Introduction}

Fetal Heart Rate (FHR) analysis has become a widely accepted means of monitoring fetal status. Currently, Doppler ultrasound and FECG have proven to be reliable techniques for monitoring FHR. The disadvantages of Doppler ultrasound systems require intermittent repositioning of the transducer and they are only suitable for use with highly trained midwifes. The use of Doppler ultrasound (non invasive manner) is not suitable for long periods of fetal heart rate monitoring [1]. In contrast, methods utilizing the abdominal electrocardiogram (AECG) have a greater prospect for long-term monitoring of FHR and fetal well being using signal processing techniques [2]. The fetal ECG is an electrical signal that can be obtained non-invasively by applying a pair of electrodes to the abdomen of a pregnant woman [3]. Sometimes the FECG is the only information source in early stage diagnostic of fetal health and status. The characteristics of the FECG, such as presence of signal, rate, waveform and dynamic behavior are useful in determining the fetal life, fetal maturity and existence of fetal distress or congenital heart disease. Therefore, the extraction of FECG signals from the abdominal ECG signal with powerful and advance methodologies is be- coming a very important requirement in biomedical engineering. The ultimate reason for the interest in FECG signal analysis is in clinical diagnosis and biomedical applications. The fetal ECG contains potentially valuable information that could assist clinicians in making more appropriate and timely decisions during labour, but the FECG signal is vulnerable to noise and difficulty of processing it accurately without significant distortion has impeded its use [4]. A number of difficulties and complication are associated with recording the abdominal ECG. Electrical activity recorded from the maternal abdomen suppresses the FECG (the magnitude of the FECG signal at the maternal abdomen is of the order of several microvolts), which is a fraction of the MECG amplitude recorded at the maternal abdomen. The abdominal ECG contains a weak fetal ECG signal, a relatively sound maternal ECG, maternal muscle noise (electromyographic activity in the muscles of the abdomen and uterus) and respiration, mains coupling, and thermal noise from the electronic equipment (electrodes, amplifiers, etc.), power line interference $(\mathrm{A} / \mathrm{C})$ and Baseline Wandering (BW). The signal processing algorithm needs to remove the maternal ECG complexes, reduce the effects of motion artifact, muscle noise and power line interface and then enhance the fetal QRS complexes before 
they can be consistently detected. Therefore, to get proper information of the fetal status and condition, it is necessary to improve the SNR of the abdominal signal. The MECG signal is the most predominant interfering signal with FECG in the abdominal signal. The frequency spectrum of each noise source partially overlaps that of the FECG and therefore filtering alone is not sufficient to achieve adequate noise reduction. Techniques to get better FECG signal acquisition remain the subject of on going research.

Although there are still limitations for extracting the FECG signal from the abdominal ECG to monitor the fetal status perfectly, currently, there is a significant amount of effort being done to improve SNR of fetal ECG signal. Conventional system reconstruction algorithms have various limitations and considerable computational complexity and many show high variance. Up to date advances in technologies of signal processing and mathematical models have made it matter-of-fact to develop advanced FECG extraction and analysis techniques. Ranges of mathematical techniques and Artificial Intelligence (AI) have acknowledged comprehensive attraction. Mathematical models incorporate wavelet transform, time-frequency approaches, Fourier transform, statistical signal analysis and higher order statistics. AI approaches towards signal recognition include Artificial Neural Networks (ANN) [5], Self-Organizing Map (SOM) neural network [6], Finite Impulse Response (FIR) neural network [7] and fuzzy logic system [8] a new technique combining the adaptive noise canceller and adaptive signal enhancer in a single recurrent neural network has been anticipated for the processing of abdominal ECG signal [9].

In the field of fetal ECG extraction, various research efforts have been carried out, including subtraction of an averaged pattern, matched filtering, adaptive filtering, orthogonal basis functions, fractals, temporal structure, frequency tracking, polynomial networks, wavelets, and real-time signal processing. Methods in fetal ECG for extracting abdominal fetal ECGs have been recently introduced for the monitor of fetal heart rate. So far, research and extensive works have been made in the area, developing better algorithms, upgrading existing methodologies, improving detection techniques to reduce noise and acquire accurate FECG signals to obtain reliable information about the fetus state thus assuring fetus well-being during pregnancy period. A. K. Barros, et al. (2001) discovered a semi-blind source separation algorithm to solve the fetal ECG extraction problem [10]. This algorithm requires a priori information about the autocorrelation function of the primary sources, to extract the desired signal (FECG). They did not assume that the sources to be statistically independent but they assumed that the sources have a temporal structure and have different autocorrelation functions. The main problem with this method is that if there is fetal heart rate variability, as is the case when the fetus is not healthy, the a priori estimate of the autocorrelation function of the fetal ECG may not be appropriate for the monitoring of the fetal heart rate.

To enhance the extraction of FECG signal from the abdominal ECG signal, in this paper, the NN-based FECG extraction has been proposed. As the neural network is adaptive to the nonlinear and time-varying features of ECG signal therefore, the neural network has been used to extract the FECG signal. Here, the adaptive linear neural network has been considered with single neuron. The input signal is considered as maternal ECG and the target signal is abdominal ECG. Using this neural network approach, the maternal ECG has been suppressed from the abdominal ECG (maternal and fetal ECG) by correlation detraction, so that the output can be considered as only fetal ECG. Therefore, this paper is paying attention for the accurate extraction of FECG signal so that the correct decision can be made by the clinician for well being of fetal during the pregnancy.

\section{Factors Affecting the Abdominal ECG Signal}

The main source of interference is the maternal electrical activity, the amplitude of which is much higher than the amplitude of the fetus electrical activity, which is often completely masked by the former. Beside this, the FECG signals are often obscured by electrical noise from other sources. Common ECG noise sources, such as power line interference, muscle contractions, respiration, skin resistance interference, instrumental noise, in addition to electromyogram and electrohysterogram due to uterine contractions, can corrupt FECG signals significantly [11]. The shape and structure of the FECG signal also depends on the placement of the electrodes although there is no standard electrode positioning for optimal FECG acquisition [12]. All of the aforementioned constraints make the FECG extraction a difficult process. Therefore, it is important to understand the characteristics of the electrical noise. Electrical noise, which will affect FECG signals, can be categorized into the following types:

MECG Signal: Maternal ECG is the most predominant interfering signal with FECG in the abdominal signal. The frequency spectrum of this noise source partially overlaps that of the ECG and therefore filtering alone is not sufficient to achieve adequate noise reduction.

Electrode Contact Noise: Electrode contact noise is transient interference caused by loss of contact between the electrode and skin, which effectively disconnects the measurement system from the subject. Electrode contact noise can be modeled as a randomly occurring rapid baseline transition, which decay exponentially to the baseline value and has a superimposed $60 \mathrm{~Hz}$ component. The transition may occur only once or may rapidly occur several times in succession. 


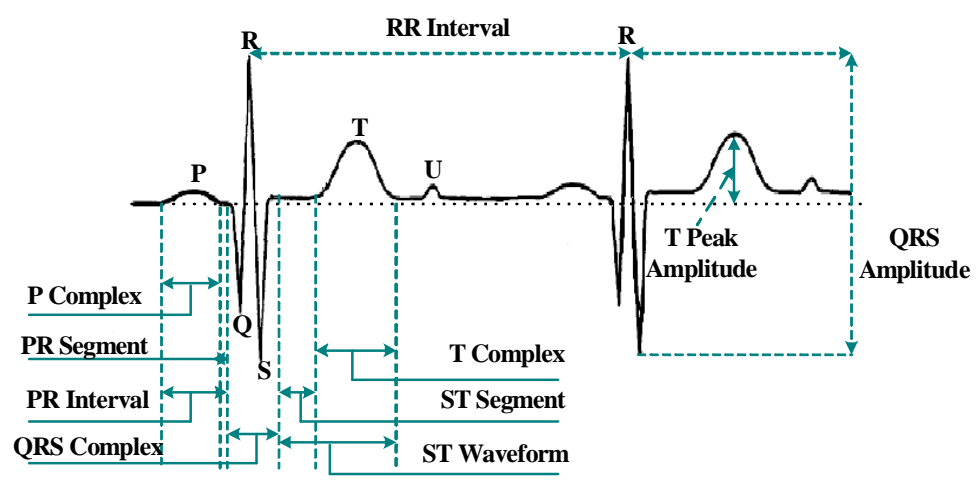

Figure 1. QRS complex in FECG signal

Motion Artifact: When motion artifact is introduced to the system, the information is skewed. Motion artifact causes irregularities in the data. There are two main sources for motion artifact, Electrode interface and Electrode cable. Motion artifact can be reduced by proper design of the electronics circuitry and set-up.

Inherent Noise in Electronics Equipment: All electronic equipments generate noise. This noise cannot be eliminated; using high quality electronic components can only reduce it.

Ambient Noise: Electromagnetic radiation is the source of this kind of noise. The surfaces of the human bodies are constantly inundated with electric-magnetic radiation and it is virtually impossible to avoid exposure to ambient noise on the surface of earth.

\section{Clinical Importance of FECG Morphology}

Biomedical signal means a collective electrical signal acquired from any organ that represents a physical variable of interest where the signal is considered in general

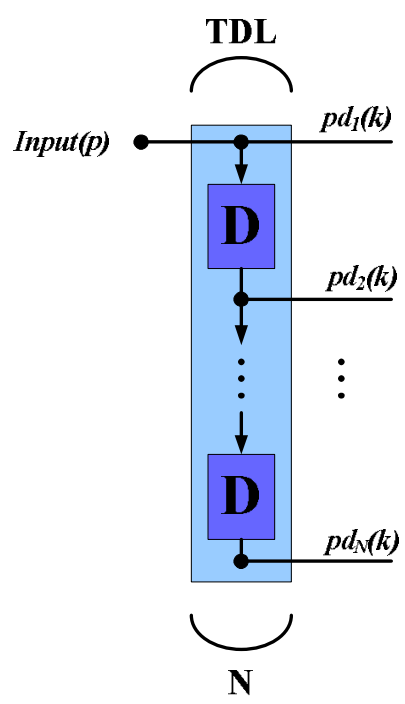

Figure 2. Tapped delay line a function of time and is describable in terms of its amplitude, frequency and phase. FECG is a biomedical signal that gives electrical representation of fetal heart rate to obtain the vital information about the condition of the fetus during pregnancy and labor from the recordings on the mother's body surface. The FECG signal is a comparatively weak signal (less than 20 percent of the mother ECG) and often embedded in noise. The fetal heart rate lies in the range from $1.3 \mathrm{~Hz}$ to $3.5 \mathrm{~Hz}$ and sometimes it is possible for the mother and some of the fetal ECG signals to be closely overlapping. The FECG is very much related to the adult ECG shown in Figure 1, containing the same basic waveforms including the Pwave, the QRS complex, and the T-wave.

The PQRST complex as shown in Figure 1 it is composed of three parts: The P-wave reflects the contraction of the atriales. Secondly, the QRS-complex is associated with the contraction of the ventricles. Due to the magnitude of the R-wave, it is extremely reliable. Finally, the $\mathrm{T}$-wave, which corresponds to the repolarisation phase which follows each heart contraction. The delay associated to the R-R interval leads to the heartbeats frequency.

\section{Methodology: Neural Network Architecture}

The architecture of the neural network is mainly designed by using the adaptive filtering approach that is the combination of ADALINE (adaptive linear network) and TDL (Tapped Delay Line). According to the concept of TDL, the input signal (maternal ECG) enters and passes through the N-1 delays and the output of the TDL is an $\mathrm{N}$-dimensional vector, made up of the input signal at the current time, the previous signal, that is fed to the ADA LINE shown in Figure 2. For the less complexity, the value of $\mathrm{N}$ is considered 2. By combination of the TDL and ADALINE network the adaptive filter network shown in Figure 3. The maternal ECG, which is predicted and closely to the abdominal ECG, passes through the 1 tapped delay line and the delayed output was multiplied by the two corresponding initial weights. After addition of the weighted output, it passes through the linear 


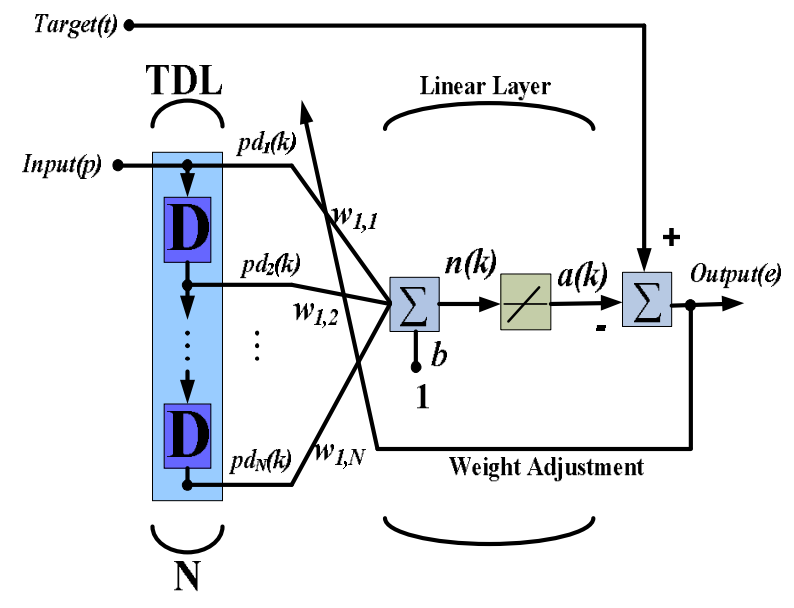

Figure 3. Adaptive filter network

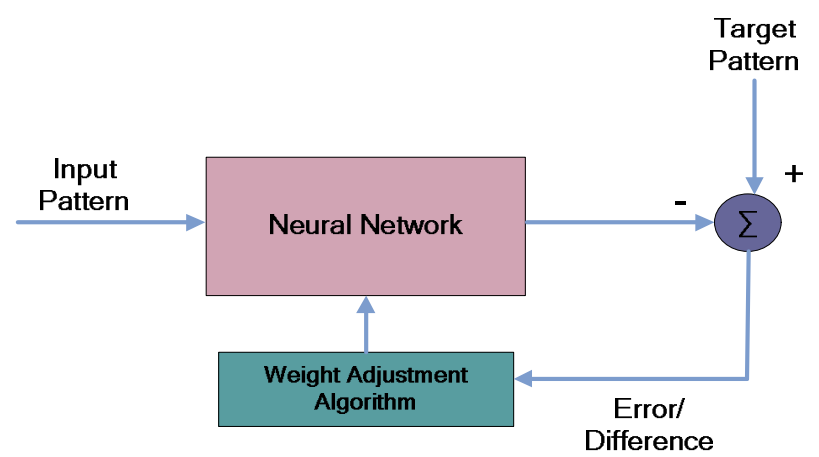

Figure 4. Neural network architecture with weight adjustment activation function. Finally, the output of the network was detracted from the target input (abdominal ECG) and to reduce the difference between input and target signal the weight has been updated every step. Therefore, the difference is considered the Fetal ECG as the abdominal ECG contains the maternal and fetal ECG, and the maternal ECG has been suppressed from the abdominal ECG. The overall architecture of the neural network is shown Figure 4, where, the difference between the target and input is error that is considered the expected outcome and by using the error, the weight has been updated.

\section{Result and Discussion}

The initial weight was considered $\mathrm{w} 1,1=0$, and $\mathrm{w} 1,2=$ -2. For input signal, 1000 data was fed into the network that is considered the maternal signal and for the target signal also 1000 data has been used as abdominal signal. Initially, the learning rate and momentum has been taken arbitrary. The changing of the learning rate and the momentum also affect the output of the network. According to the out put it has been observed that the learning rate is low that time the fetal signal was not reasonable but the increased value of the learning rate, the suppressed output from the target signal that is the fetal ECG was very much satisfactory. Again, the effects of moments also observed. If the low momentum value used in the network that time the fetal signal contains some unwanted signal. Therefore, the high learning rate $=1$, and the low momentum value $=0.2$ has been considered to get the maximum satisfactory fetal ECG output that is shown in Figure 5. According to the figure, at around the

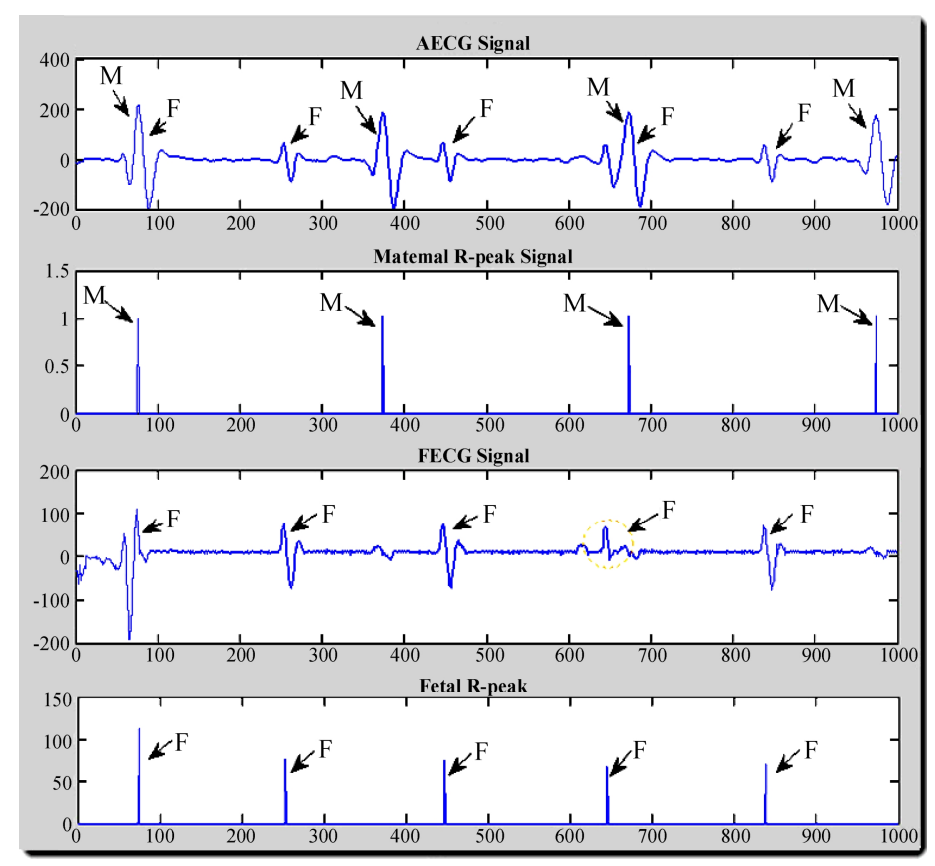

Figure 5. Suppressed fetal ECG from abdominal ECG to maternal ECG 


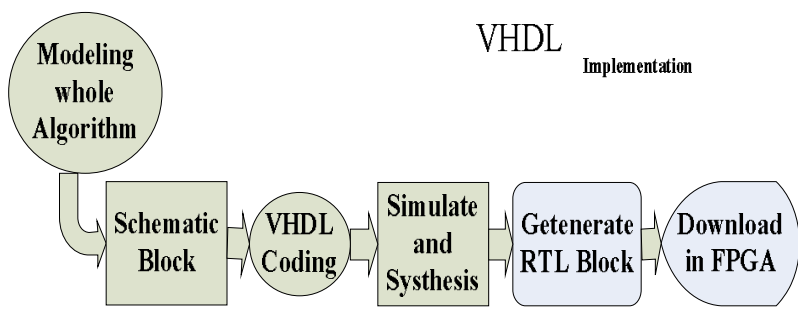

Figure 6. Overall system architecture

100 sample data, the QRS of the FECG signal is not clear like the ordinary FECG QRS; sometimes it can be also reasonable by changing the appropriate value of the learning rate and the value of the momentum. Because, it has been observed that the FECG signal after extracting from the abdominal ECG to maternal ECG is greatly affected by the value of learning constant and momentum.

\section{Future Works}

The work described here is currently on-going and is part of a larger approach incorporating this utility. The developed algorithm is with the objective of implementing in hardware. Therefore, the future work will involve modeling the algorithm with VHDL and perform simulation and synthesis for hardware implementation and download into FPGA (Field Programmable Gate Array). The overall system architecture for hardware implementation has shown in Figure 6.

\section{Conclusions}

FECG signal contains the valuable information that could assist clinicians in making more appropriate and timely decisions. The technique, adaptive neural network filtering approach has been used to extract the FECG signal from the abdominal ECG that is the agreeable output. In brief, the accuracy of output depends on how many variations of signals are used as input and the target in the network. Furthermore, in this approach, learning rate and momentum is also an important factor to affect the desired FECG signal. By the observation the technique is working high learning rate and low momentum value.

\section{Acknowledgments}

The authors would like to express sincere gratitude to the Ministry of Science, Technology and Innovation of Malaysia for providing fund for the research under eScienceFund grant (Project No.01-01-08-SF0029).

\section{REFERENCES}

[1] M. Ungureanu, J. W. M. Bergmans, M. Mischi, S. G. Oei, and R. Strungaru, "Improved method for fetal heart rate monitoring," Proceedings of the 2005 IEEE Engineering in Medicine and Biology $27^{\text {th }}$ Annual International Conference, Shanghai, China, pp. 5916-5919, January 2006.

[2] P. P. Kanjilal, S. Palit, and G. Saha, "Fetal ECG extraction from single-channel maternal ECG using singular value decomposition," IEEE Transactions on Biomedical Engineering, Vol. 44, pp. 51-59, 1997.

[3] T. Solum, I. Ingermarsson, and A. Nygren, "The accuracy of abdominal ECG for fetal electronic monitoring," Journal of Perinatal Medicine, Vol. 8, No 3, pp. 142-149, 1980 .

[4] K. Karlsso, H. Lilja, K. Lindecrantz, and K. G. Rosen, "Microprocessor based waveform analysis of the fetal electrocardiogram during labor," International Journal of Gynaecol and Obstetrics, Vol. 30, No. 2, pp. 109-16, 1989.

[5] M. B. I. Reaz and L. S. Wie, "Adaptive linear neural network filter for fetal ECG extraction," Proceedings of International Conference on Intelligent Sensing and Information Processing, Chennai, India, pp. 321-324, January 2004.

[6] G. Vasios, A. Prentza, D. Blana, E. Salamalekis, and P. Thomopoulos, "Classification of fetal heart rate tracings based on wavelet transform and self organizing map neural networks," Proceedings of the $23^{\text {rd }}$ Annual International conference of the IEEE, Istanbul, Turkey, Vol. 2, pp. 1633-1636, 2001.

[7] G. Camps, M. Martinez, and E. Soria, "Fetal ECG extraction using an FIR neural network," Computers in Cardiology, Rotterdam, Netherlands, pp. 249-252, 2001.

[8] K. A. K. Azad, Z. M. Darus, and M. A. M. Ali, "Development of a fuzzy rule-based QRS detection algorithm for fetal and maternal heart rate monitoring," Proceedings of the $20^{\text {th }}$ Annual International Conference of the IEEE Engineering in Medicine and Biology Society, Vol. 1, pp. 170-173, 1998.

[9] S. Selvan and R. Srinivasan, "A novel adaptive filtering technique for the processing of abdominal fetal electrocardiogram using neural network," Adaptive Systems for Signal Processing, Communications, and Control Symposium 2000. (AS-SPCC), Louise, Alta., Canada, pp. 289-292, 2000

[10] A. K. Barros and A. Cichocki, "Extraction of specific signals with temporal structure," Neural Computation, MIT Press, Vol. 13, pp. 1995-2003, 2001.

[11] V. Zarzoso, A. K. Nandi, and E. Bacharakis, "Maternal and foetal ECG seperation using blind source separation methods," IMA Journal of Mathematics Applied in Medicine and Biology, Vol. 14, pp. 207-225, 1997.

[12] F. Vrins, C. Jutten, and M. Verleysen, "Sensor array and electrode selection for non-invasive fetal electrocardiogram extraction by independent component analysis," Proceedings $5^{\text {th }}$ International Conference Independent Component Analysis, Granada, Spain, pp. 1017-1024, 2004. 